\title{
Classification based on Predictive Association Rule for Discrimination Prevention
}

\author{
Ankita K. Shinde, Pramod B.Mali \\ Department of Computer Engineering, \\ STES's Smt. Kashibai Navale College of Engineering, \\ Pune - 411041, India
}

\begin{abstract}
In data mining, discrimination is the subject which has been extensively studied in social and economic science. However, there are negative perceptions about data mining. Discrimination comes under two categories one is direct and second is indirect. Decisions based on sensitive attributes are termed as direct discrimination and the decisions which are based on non-sensitive attributes are termed as indirect discrimination which is strongly correlated with biased sensitive once. There are many new techniques proposed for solving discrimination prevention problems by applying direct or indirect discrimination prevention individually or both at the same time. New metrics to evaluate the utility were proposed and are compared with approaches. The proposed work discusses how privacy preservation and prevention between discrimination is implementing with the help of post processing approach. The Classification based on predictive association rules (CPAR) is a kind of association classification methods which combines the advantages of both associative classification and traditional rule-based classification which is used to prevent discrimination prevention in post processing by improving accuracy.
\end{abstract}

\section{Keywords}

Data-mining, Discrimination, DRP, Classification using predictive association rule (CPAR)

\section{INTRODUCTION}

The word discrimination originates from the Latin discriminare, which means to distinguish between. In social and economic mean discrimination is unfair treatment of people on the basis of their category. At research side, the discrimination has become an issue in credit, mortgage, insurance, labor market, education and other human activites and has attracted much interest of researcher in economics and social science.

There are several decision-making tasks which lend themselves to discrimination, e.g. loan granting, education, health insurances and staff selection. In many scenarios, decision-making tasks are supported by information systems. Given a set of information items on a potential customer, an automated system decides whether the customer is to be recommended for a credit or a certain type of life insurance. Automating such decisions reduces the workload of the staff of banks and insurance companies, among other organizations. The use of information systems based on data mining technology for decision making has attracted the attention of many researchers in the field of computer science. In consequence, automated data collection and a massive amount of data in data mining techniques such as association/classification rule mining have been designed and are currently widely used for making automated decisions. At first sight, automating decisions may give a sense of fairness: classification rules (decision rules) do not guide themselves by personal preferences. However, at a closer look, one realizes that classification rules are actually learned by the system based on training data. If the training data are inherently biased against particular community (for example, foreigners), the learned model may show a discriminatory behavior. For e.g. If a company wants to hire those people who don't know Java from their online job profile management system then it is an unfair decision. The people cannot be discriminated on the basis of IT programming language (e.g. Java) because IT programming languages are having their sub-classes also (Java-JSP, Java-Servlet). If the original biased dataset DB is used for data analysis without any anti-discrimination process (i.e. discrimination discovery and prevention), the discriminatory rules extracted could lead to automated unfair decisions. On the contrary, DB can go through an antidiscrimination process so that the learned rules are free of discrimination. As a result, fair and legitimate automated decisions are enabled. Discrimination can be either direct or indirect Direct discriminatory rules indicate biased rules that are directly inferred from discriminatory items (Indirect discriminatory rules (redlining rules) indicate biased rules that are indirectly inferred from non-discriminatory items because of their correlation with discriminatory ones. Indirect discrimination could happen because of the availability of some background knowledge (rules), for example, indicating that a certain zip code has become progressively worse in area. The background knowledge might be accessible from publicly available data (e.g. census data) or might be obtained from the original dataset itself because of the existence of non-discriminatory attributes that are highly correlated with the sensitive ones in the original dataset.

Discrimination prevention consists of inducing patterns that do not lead to discriminatory decisions even if the original training data sets are biased. Three approaches are possible:

- $\quad$ Preprocessing

- Postprocessing

- Inprocessing

In this paper, we are going to prevent the discrimination on preprocessing and postprocessing.

In data mining and machine learning [7], various classification models are constructed on the basis of historical data exactly with the purpose of discrimination by distinguishing between elements of different classes. Although there were some new methods have recently been proposed for discrimination prevention. However, such works only consider direct and indirect discrimination with preprocessing approach. Their approaches cannot guarantee that the transformed dataset is really discrimination-free, because it is known that discriminatory behaviours can be hidden behind nondiscriminatory items. 


\section{MOTIVATION}

As it has been known that discrimination is big issue in data mining.Inorder to tackle the discrimination problem various new techniques were proposed using direct and indirect discrimination prevention. For solving direct and indirect discrimination prevention preprocessing approach was proposed.Preporocessing approach consider being more flexible one [8], it does not require changing the standard data mining algorithm. But this approach cannot guarantee that the transformed dataset is discrimination free and could not solve direct discrimination.

Therefore inorder to make dataset discrimination free various other algorithms were proposed [4]. There are many algorithms in preprocessing like Direct Rule Protection, Direct Rule Generalization, Direct and Indirect Rule Protection which is used inorder to make dataset discrimination free [1]. But still it lack in its efficiency and accuracy. Therefore, the algorithm one which is considering in this paper is post processing approach for removing direct and indirect discrimination from the dataset. This leads to think of using various other approaches like Inprocessing, postprocessing out of which post processing approach which is a challenging issue for maintaining data quality and reduce the data loss. So using algorithm like CPAR with post processing approach data quality can be maintain without much data loss. Therefore to the best of our knowledge this will be the first work that proposes the post processing approach for direct and indirect discrimination prevention.

\section{RELATED WORKS}

The discrimination problem in data mining model was addressed in [6]. Also how discrimination may be hidden in data mining models was considered. But still the issue of antidiscrimination in data mining didn't receive much attention. The discovery of discriminatory decisions was first proposed by Pedreschi [2], the initial idea of anti-discrimination for cyber security application based on data mining using rule protection and rule generalization for direct discrimination prevention was introduced. The use of rule protection in a different way for preventing indirect discrimination in a dataset was introduced [3]. Discrimination measures for classification rules was introduced [5] were a systematic framework for measuring discrimination, based on the analysis of the historical decision records stored out of a socially-sensitive decision task, e.g., credit approval. A notion of statistical significance was included and combined with the discrimination measures with association rule mining to work with direct and indirect discrimination in datasets of decisions for rule-based classifiers. Preprocessing discrimination prevention methodology including different data transformation methods that can prevent direct discrimination, indirect discrimination or both of them at the same time was proposed [1].

In the existing System [1], various approaches were used for solving discrimination problem like preprocessing, Inprocessing and post processing approach. Out of these approaches preprocessing was chosen to be more flexible one to solve the direct discrimination issue in data mining. Here the Apriori algorithm has used to find the FIS (Frequent Item Dataset).Once this FISs are obtained they are used for further processing to find the PD and PND rules. In [1], PD rules are then again further classified into Alpha protected and Alpha Discriminated. If its Alpha discriminated then DRP algorithm has been used to make remove discriminatory attributes from the dataset. Similarly PND rules are further classified into redlining and Non-redlining rules. If non-redlining rules are found they are further processed by indirect algorithm to make the PND rules redlining rules and remove the discriminated attributes indirectly. Also new preprocessing discrimination prevention methodology including different transformation methods were prevent with direct and indirect discrimination and both at the same time. On the other hand, the impact of the method in terms of information loss (i.e., data quality loss) is not properly maintained. The proposed system uses discrimination prevention in Postprocessing approach were the resulting data mining model is modified instead of cleaning the original data set or changing the data mining algorithm. However, we are implementing CPAR algorithm which is used to prevent discrimination prevention in postprocessing.

Using Classification based on predictive association rules (CPAR) in data mining discrimination prevention can be prevent using post processing approach. In Postprocessing the resulting data mining model is modified instead of cleaning the original data set or changing the data mining algorithm. As it is a challenging approach we can prevent discrimination by removing discriminatory attributes from datasets. To obtain FR (frequent Result) the CPAR algorithm will be use, which uses association rule mining algorithm, such as Apriori or FPgrowth, to generate the complete set of association rules and achieve higher classification accuracy than traditional classification approaches. It combines the advantages of both associative classification and traditional rule-based classification.

\section{PROPOSED WORK}

The proposed solution to prevent direct discrimination is based on the fact that the data set of decision rules would be free of direct discrimination if it only contained PD rules that are protective or are instances of at least one non-redlining PND rule. Therefore, a suitable data transformation with minimum information loss should be applied in such a way that each discriminatory rule either becomes $\alpha$ protective or an instance of a non-redlining PND rule. We call the first procedure direct rule protection (DRP) and the second one rule generalization.

The proposed techniques should be evaluated based on two aspects. One way, we need to measure the success of the method in removing all evidence of direct and/or indirect discrimination from the original data set; on the other hand, we need to measure the impact of the method in terms of information loss (i.e., data quality loss).

Our algorithms based on the direct and indirect discrimination prevention methods Proposed. There are some assumptions common to all algorithms in this section. First, we assume the class attribute in the original data set DB to be binary. Second, we consider classification rules with negative decision to be in FR.Third, we assume the discriminatory item sets and the nondiscriminatory item sets to be binary and non-binary categorical. Start with direct rule protection and Indirect Rule protection.

Even though preprocessing approach is easy and very flexible to remove discrimination from the dataset still it would cause much information loss. Therefore postprocessing approach is used in order to maintain data quality and improve the accuracy.

The application developed is based on dataset. The dataset is provided by a user who wants discrimination protected dataset. For this application, IT Company is being considered 


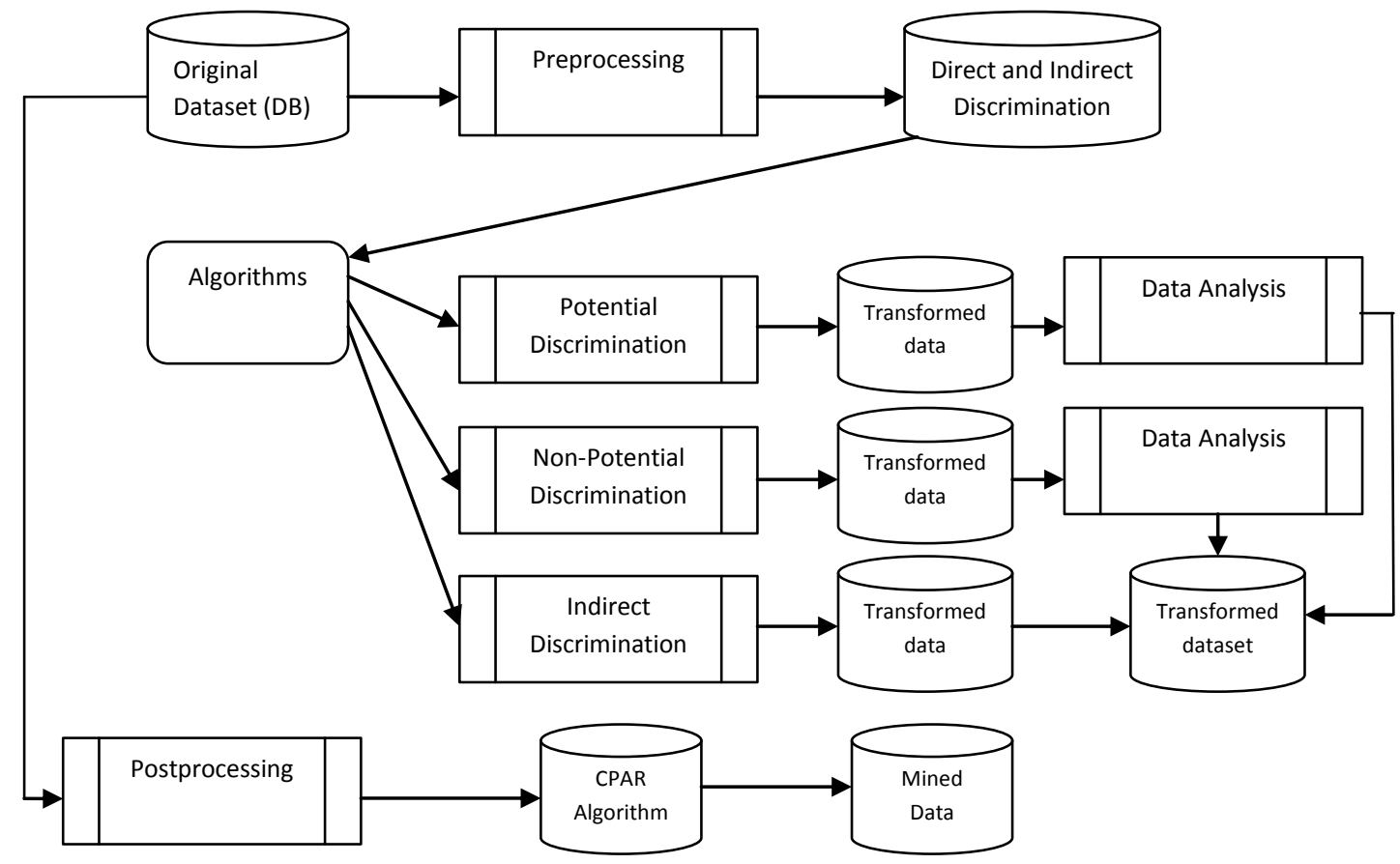

Fig.1 Block Diagram of Discrimination processing for Pre-Processing and Post-Processing

based dataset. The applicants who wants job in that company will maintain their job profile and select the languages in which they prefer to work. Now, the company will decide the classification attribute and discriminated attribute. On this context, the company will hire the people and the application's job is to prevent the discriminated rules which can be done through post processing and preprocessing.

Fig. 1 shows the block diagram of discrimination processing for preprocessing and postprocessing approach.Here the objective is to reduce the information loss by maintaining the data quality in the proposed application. For performing this, we are using transformation techniques and utility measures and algorithms like Direct Rule Protection, Direct Rule Generalization, Direct and Indirect Discrimination Prevention and CPAR algorithm. Out of which CPAR is more efficient than remaining algorithms in terms of information loss and improving the accuracy of algorithm.

\section{ALGORITHM DISCRIPTION}

The main idea of this paper is to implement CPAR algorithm. As CPAR algorithm is more efficient then FOIL (First Order Inductive Learner) and PRM (Predictive Rule Mining) algorithms, the basic difference in these strategies is in rule generation process. Foil generates rules which are not redundant but to achieve this, it loses some important rules. So PRM extracted these rules also but with cost of redundancy. Some rule may be extracted more than ones. CPAR also uses similar concept of PRM as to generate more rule with some redundant rules, but it can test more than one attribute at a time to judge whether this attribute can also give some useful rule or not. So more rules and less computation is needed in CPAR for comparison to the PRM algorithm. To implement these algorithms, following three steps are used:

1. Generation of rule.

2. Estimate Accuracy of rules.

3. Classification and Result analysis.
The main difference between CPAR and PRM is that instead of choosing only one attribute to obtain best gain on each iteration(as in FOIL and PRM),CPAR choose a number of attributes if those attributes have similar best gain. This is done by applying GAIN_SIMILARITY_RATIO and by calculating the minimum gain.

CPAR takes input as (space separated) binary valued dataset $\mathrm{R}$ and produces a set of CARs. It also requires minimum gain constant which is user defined value, decay factor and TOTAL_WEIGHT_THRESHOLD. The resulting data is in the form of linked-list of rules ordered according to Laplace accuracy.

\section{CONCLUSION}

As discrimination is a very important issue of data mining. The purpose of this paper was to develop a new preprocessing discrimination prevention including different data transformation methods that can prevent direct discrimination, indirect discrimination along with both at the same time. As future work we are exploring measures of discrimination different from the ones considered in this paper along with privacy preservation in data mining. Further, implementation of discrimination prevention in Postprocessing will be done. CPAR achieves high accuracy and efficiency, which can be credited to the following distinguished features: First, it uses greedy approach in rule generation, which is much more efficient than generating all candidate rules. Second, it uses a dynamic programming approach to avoid repeated calculation in rule generation. Third, it selects multiple literals and builds multiple rules simultaneously. Fourth, it uses expected accuracy to evaluate rules, and uses the best $\mathrm{k}$ rules in prediction. CPAR represents a new approach towards efficient and high quality classification However, by implementing CPAR algorithm which shows better efficiency than FOIL and PRM and use to prevent discrimination prevention in postprocessing. 


\section{REFERENCES}

[1] Sara hajian and Josep Domingo-Ferrer, Fellow, IEEE, A Methodology For Direct and Indirect Discrimination Prevention in Data Mining, Proc.IEEE Transaction on Knowlegde And Data Engineering,vol.25,no.7,July 2013.

[2] S. Hajian, J. Domingo-Ferrer, and A. Martinez-Balleste, Discrimination Prevention in Data Mining for Intrusion and Crime Detection, Proc. IEEE Symp. Computational Intelligence in Cyber Security (CICS 11), pp. 47-54, 2011.

[3] S. Hajian, J. Domingo-Ferrer, and A. Martinez-Balleste, Rule Protection for Indirect Discrimination Prevention in Data Mining, Proc. Eighth Intl Conf. Modelling Decisions for Artificial Intelligence (MDAI 11), pp. 211222 , and 2011.
[4] F. Kamiran and T. Calders, Classification without Discrimination, Proc. IEEE Second Intl Conf. Computer, Control and Comm. (IC4 09), 2009.

[5] D. Pedreschi, S. Ruggieri, and F. Turini, Measuring Discrimination in Socially Sensitive Decision Records, Proc. Ninth SIAM Data Mining Conf. (SDM 09), pp. 581-592, 2009.

[6] D. Pedreschi, S. Ruggieri, and F. Turini, DiscriminationAware Data Mining, Proc. 14th ACM Intl Conf. Knowledge Discovery and Data Mining (KDD 08), pp. 560-568, 2008

[7] P.N. Tan, M. Steinbach, and V. Kumar, Introduction to Data Mining. Addison Wesley, 2006.

[8] R. Agrawal and R. Srikant, Fast Algorithms for Mining Association Rules in Large Databases, Proc. 20th Intl Conf. Very Large Data Bases, pp. 487-499, 1994 